\title{
Strategies of the Level-By-Level Approach to the Minimal Route
}

\author{
Nikolay Starostin ${ }^{*}, 1$, Konstantin Mironov ${ }^{1,2}$ \\ ${ }^{1}$ Institute of New Materials and Technologies, Ural Federal University, 620002, Russia \\ ${ }^{2}$ Faculty of Computer Science and Robotics, Ufa State Aviation Technical University, 450008, Russia
}

\begin{tabular}{l} 
A R T I C L E I N F O \\
\hline Article history: \\
Received: 20 December, 2018 \\
Accepted: 07 February, 2019 \\
Online : 20 February, 2019 \\
\hline Keywords: \\
Optimization \\
Minimum route \\
Traveling salesman problem \\
TSP \\
CNC \\
\end{tabular}

\begin{abstract}
A B S T R A C T
The task of optimal path planning for drilling tool with numerical control is considered. Such tools are used in the production of printed circuit boards. The algorithm modification of level-by-level route construction for the approximate solution of the traveling salesman problem is discussed. Bypass objects can be specified either as a table of distances or values represented by a symmetric matrix, or as Cartesian coordinates (in applied cases of using numerically controlled equipment). The algorithm was tested on many different examples. As a result of the calculations performed for examples from the TSPLIB library (defined through a full distance matrix or Cartesian coordinates) with a dimension of up to 100 cities, the ability of the algorithm to construct the optimal route was confirmed. For examples from other sources or for artificially constructed ones (up to 130 objects), in the testing of the algorithm, the declared record values of the route minimum length were also achieved or improved.
\end{abstract}

\section{Introduction}

This paper is an extension of the article presented at the IEEE International Symposium on Signal Processing and Information Technology [1]. Initially, the problem of the rapid acquisition of an optimal route for moving the tool between processing zones on computer numerical control (CNC) equipment was considered, which is close in form to the symmetric traveling salesman problem with the Euclidean metric. For a limited number of processing zones, a solution [2, 3] belonging to the category of composite methods according to classification [4] was proposed. Construction of a convex hull, use of branch and boundary method elements, level-by-level analysis and route options design. This made it relatively easy to synthesize the optimal result for a small number of processing objects. Subsequently, the need to increase the effectiveness of this approach was revealed, where an important role, just like in the branch and boundary method, is played by a successful choice of upper bounds for the solution for which heuristic algorithms are used [5].

In the process of developing the required approximate algorithm, it was natural to use state of the art techniques from the exact method [3], while somewhat broadening the general formulation of the problem. Namely (in terms of the traveling salesman problem), to find the shortest closed route passing

${ }^{*}$ Corresponding Author: Konstantin Mironov, mironovconst@gmail.com through $\mathrm{n}$ cities, visiting each city exactly once. The distance between pairs of cities is determined by a symmetric, not metric in the general case, matrix of real numbers $C=\|c i, j\|$. In [6] a natural version of the transition to the approximate method of searching for the minimum route in this formulation is presented, while the basic procedure, the basic calculation schemes and possible directions of development are described. In [7 - 9], the calculation procedures are detailed and studied, the results of testing are given, along with options for constructing the algorithm based on different schemes from the combined levels. For all benchmark tests (examples from [10] up to 100 cities inclusive, given by a complete table of distances or by Cartesian coordinates), optimal routes are obtained. Also, for all of several dozen random tests, with reported results (dimension in the range of 30-60 cities), for example, as in [11], record values of the minimum length of the route were reached, or these figures improved.

\section{Basic procedure and trivial calculation scheme}

If we rely on the algorithms' classification from [12], then the level-by-level approximation (considered and tested in [7,9], respectively, for the matrix and coordinate representation) can be attributed to the symbiosis of "tour construction algorithms" and "monotonic algorithms for improving the tour." Specifically, such one when the procedure for identifying improvements to the tour is involved at different levels of the multi-stage building process. According construction details, the proposed algorithm is closest 
to a truncated combination of three algorithms from the classification [12]. This is $\mathbf{C}$ (arbitrary connection). "Let $\left(i_{1}, i_{2}\right.$, $\ldots, i_{n}$, be an order of vertices. Form a two-member tour $\left(i_{1}, i_{2}\right)$. The vertex $i_{l}(l=3,4, \ldots n)$ is to be included in the $(l-1)$-segment (pod) in such a place that the increase in the tour ... is minimal». CR (most remote connection). "Act like in $C$. At step $l(l=3,4$, ... n) in the (l-1) -st tour include the vertex $i_{l}$, for which the minimum distance to already included vertices is the largest among all vertices not yet included». CE (most economic connection). «Act like in C. At step $l(l=3,4, \ldots n)$ in the (l-1) -st tour include the vertex $i$, for which the increase in the tour is minimal among all the vertices not yet included. However, this combination emphasizes somewhat different points. It should also be noted that the proposed algorithm is multi-pass. Conscious departure from the seemingly natural use of coordinates for $\mathrm{CNC}$ equipment should also be underscored. This is done because different metrics can be used for different types of equipment and, accordingly, of technological processes (calculations of distances between treatment zones), therefore for the sake of greater generality we will not rely on the advantages of using metric space.

The essence of the basic algorithm is as follows. Let i1, i2,..., in be a certain order of processing zones, vertices or cities in terms of the traveling salesman problem, where for definiteness i1 and i2 are the most distant cities from each other. Build a tour from i1 to i2 and back, i.e., a two-member closed tour (i1, i2, i1). Next, include the city ik $(\mathrm{k}=3)$ in the existing two-member tour. This can be done in 2 ways: either (i1, ik, i2, i1) or (i1, i2, ik, i1). Since they are equivalent, we will explain the process using the first one as an example. So, for each of the two three-member tours, it is necessary to perform consecutive actions starting from the step $1=$ 4 . In step $1(1=4,5, \ldots, n)$, in each of the available $(1-1)$-member tours, include one of the remaining cities il $(1=4,5,6, \ldots, n)$ in that place of the tour, where it will provide a minimum tour increase based of the inclusion of this city. At the same time, the city of inclusion must be such that the value of the minimum increment of the tour from its inclusion is the maximum among the similar values of the minimum increments from other cities of the potential inclusion. Thus, at the last step $(1=n)$ we obtain two complete routes. To fix one of them with the smallest length (in general, their length is the same, and the routes themselves coincide, the difference is only in the direction of the detour in the forward or reverse direction). Thus, the first of the approximate routes (Hamiltonian cycle) is obtained, which corresponds to its progenitor - a three-member tour i1, ik, i2, i1. $(\mathrm{k}=3)$. Then go back to the original two-member tour (i1, i2, i1) and perform similar calculations for the remaining cities ik $(\mathrm{k}=4,5, \ldots \mathrm{n})$. As the result, we will obtain n-2 approximate route options. Each route is determined by uniform constructions from its initial threemember tour i1 , ik, i2, i1, $(k=3,4, \ldots n)$. We call the routes formed in this way from the original three-member tours, routes of level 0 and, for convenience, assign them indices $(00,01,02, \ldots)$ in accordance with the order of their length increase.

It can be noted that the level 00 route (s), with any significant number of cities, rarely reach optimum or are close enough to it. Therefore, it is required to continue similar constructions in order to obtain level 1 routes. They are similarly constructed for each of the $n-2$ initial three-member tours i1, ik, i2, i1, $(k=3,4, \ldots n)$. At the same time, at least one of the new routes (for each of $\mathrm{k}=3,4$, ...n) will be no longer by construction than its related route of level 0 .
Let us explain this calculation by the example of constructing the first of the routes for level 1. In the initial tour (i1, ik, i2, i1), (k $=3$ ), include the city im (for definiteness $\mathrm{m}=4$ ) alternately between pairs of adjacent cities. Accordingly, obtain three 4membered closed tours (i1, im, ik, i2, i1), (i1, ik, im, i2, i1), (i1, ik, $\mathrm{i} 2$, im, i1). In step $1(1=5,6, \ldots \mathrm{n})$, in each of the available $(1-1)$ -member tours, include one of the remaining cities il $(1=5,6, \ldots$, $\mathrm{n})$ in the place of the tour where a minimum tour increase will be provided from the inclusion of this city. At the same time, the city of inclusion must be such that the value of the minimum increment of the tour from its inclusion is the maximum among the similar values of the minimum increments from other cities of the potential inclusion. Thus, at the last step $(1=n)$ we get three complete routes. Fix one of them with the shortest length. This is the first of the approximate level 1 routes. Together with it, the initial 4-member tour is fixed as well (the value of $\mathrm{m}, \mathrm{k}$ and the location of the corresponding cities in the tour).

To form the remaining routes of this level (with $\mathrm{k}=3$ ), we need to return to the original three-member tour (i1, ik, i2, i1) and perform similar actions with other valid values im $(m=5,6, \ldots, n)$, as in the construction of the first route of level 1. As a result, for the value of $\mathrm{k}$ under consideration, we obtain n-3 Hamiltonian cycles, i.e., approximate routes of level 1.

To form the remaining routes of this level (n-3 for each new $k$, where $\mathrm{k}=4,5, \ldots, \mathrm{n})$, we need to return to the original threemember tours (i1, ik, i2, i1). Perform similar actions for each of $\mathrm{k}$ with admissible values of im, as in the construction of the first group of routes of level 1 . As a result, for each of $\mathrm{k}(\mathrm{k}=4,5, \ldots, \mathrm{n})$ we get n-3 full routes of level 1. All received routes in each group (fixed value $\mathrm{k}$ ) are assigned indices $(10,11,12, \ldots)$ in accordance with the order of increasing their lengths (several routes can have the same index if their lengths are equal to each other).

Similarly, routes of levels 2,3 and subsequent are built, respectively, from 4,5 etc. member source tours identified at the previous level.

But if nothing restricts this process, then we will have an avalanche-like growth of calculations. At the same time, when moving inward through the levels, a situation similar to the "thermal modeling" algorithms [12, 13], may be observed, where the process of improving the complete route is attenuated. For greater certainty, in [9], based on the accumulated statistical data, examples of different dimensionality of the level number, beyond which constructions are hardly advisable, are roughly indicated. When the level-by-level constructions are terminated according to the conditioned criterion, then in accordance with the multipass algorithm, we must again go to the two-member closed tour (i1, i2) for successive inclusion of the vertex ik $(k=4,5, \ldots n)$, as in the sample presented above (for the case $\mathrm{k}=3$ ). As a result, the route, the smallest of all constructed, is determined. It is also a potential contender for correspondence to the optimal route. This correspondence is consistently confirmed when testing the examples of a relatively small (up to 60 elements) dimension. Additionally, in practice, for achieving records from the original tripartite tours (i1, ik, i2), in tests from $[10,11,14]$ with a dimension of 25-60 vertices, it was sufficient to perform calculations not for all but only for several vertices ik where the values of index $\mathrm{k}$ correspond to those inclusion vertices that form zero-level routes with lower indices [7, 8]. In addition, for an overall reduction in the calculation of tests with a dimension of 60 100 vertices, extended schemes can be used, so that to reach a 
record we would also be limited to calculations with only lower indices from the starting extended level [9].

In general, starting from this basic procedure with a trivial calculation scheme, it is necessary to form strategies rational for practical use to create complete routes and check the criteria for stopping calculations.

\section{Possible directions in organizing calculations.}

First, we return to the problems of CNC machine tools. Drilling holes in printed circuit boards is one of two sources of TSP instances that are associated with practical application [16]. From an applied point of view, local search algorithms are best for solving the traveling salesman problem [17]. Nevertheless, practical problems of this kind can bring certain difficulties for local search, even with a small number of vertices. So, for example, one of such effective algorithms with the Lin-Kernigan neighborhood implemented in the Concord program [17], does an excellent job with large-dimension tests from [10], but gives, for example, a fragment of a printed circuit board with a dimension of 15 vertices, which is not an important result (approximately 103\% from optimum [1]). To obtain the optimal route (Figure 1 on the right), the program needs to change the default settings, which may not help at all in more complex cases. In addition, metaheuristic methods are very popular for practical problems. [18]. They do not guarantee that the best solution to the problem will be achieved, but with a successful setup they can find a "good enough" result. Their disadvantage is the presence of a different number of control parameters [20], which makes it difficult to set up algorithms for solving specific problems.

Now let us consider possible approaches for the organization of calculations in the framework of our basic algorithm for the level-based approximation.

\subsection{Calculation on the basis of not deteriorating routes}

Theoretically, each of the n-2 initial 3-member tours i1, ik, i2, $\mathrm{i} 1,(\mathrm{k}=3,4, \ldots, \mathrm{n})$, from which level 1 routes are formed, can be the progenitor of the optimal route. That, however, can no longer be said about each of the 4-member (and subsequent) tours, from which routes of level 2 and further are formed. Therefore, after constructing routes of level 1 (for each of the $n-2$ three-member tours), the route (s) with the index zero (10) is (are) allocated to each of the received $n-2$ groups and the corresponding fourmember tour is fixed for them, from which the subsequent construction will go (routes with a different level index and the corresponding components are not considered further). Level 2 routes are similarly constructed on this base (level 2), after which only route (s) with index zero (20) and so on is (are) also considered (that is, at subsequent levels everything related to nonzero indices routes is not participating). Thus, routes of non-zero level $\mathrm{J}(\mathrm{J}=2,3,4, \ldots)$ are constructed similarly to routes of level 1 , from their initial $(\mathrm{J}+2)$-fold tours, which correspond to full routes of levels $(\mathrm{J}-1) 0$. The whole process of level building can be conditionally represented by the following scheme:

\section{(1) level $0_{i}-$ level $1_{0}-$ level $2_{0} \ldots(i=0,1,2, \ldots)$}

The shortest length among all level 1 routes will be the route with a zero index (level 10), which is the result of the initial embedding of a certain city iz $(z \neq 1,2, k)$ into a certain place of the tour (i1, ik, i2, i1). By construction, its length cannot be greater than the length of the "parent" route of level 0 , resulting from embedding in the tour (i1, i2, i1) of city ik. The same applies to all derived routes with a zero index of subsequent levels, i.e., the best routes of the level cannot be longer than their ancestors. Moreover, when building at the initial levels (when moving from a lower level number to a larger number), the length of the descendant route decreases; as practice shows, this is a frequent case. However, as the level numbers increase, this happens less frequently and then fades out.

Therefore, the process of constructing routes in ascending level numbers continues, approximately as in "thermal modeling" algorithms, until a certain equilibrium is reached, understood as "no significant deviations from the found tour length are observed for a long time" $[12,13]$, i.e., until the termination of reducing the current length of the route. After that, the level constructions in this direction are interrupted. In the end (when the calculation is stopped in all available directions) the route is determined, the smallest of all constructed. It is also a potential contender for compliance with the optimal route. In $[6,7]$, the main results of testing in the framework of the considered scheme (1) for several dozens of tasks with cities given by a symmetric table of distances up to 60 objects in dimension are presented. At the same time, in all calculations, the reported record results were obtained or improved.

To explain the essence of the material presented in [1], a simple example was selected with 29 bypass objects from the TSPLIB library [10], and to demonstrate the results of innovations, a test (file xqf131.tsp) measuring 131 elements from the VLSI TSPs collection [20] created in based on SBIS data sets investigated at the University of Bonn.

Here, as a simple illustrative material, we take a classic sample containing 48 objects (att48_d.txt file) from a library with TSP data [21]. This is one of the key examples put on the cover of a famous book [22], which was used in it to illustrate existing approaches to solving the problem in question. To demonstrate the results of innovations, we will again use the same test of 131 elements.

So, let us explain the organization of the process for finding the minimum route on the selected example with 48 US cities (att48 d.txt file), given by the complete distance table. For graphical representations of the constructed routes, the Cartesian coordinates of cities from the att48.tsp [10] file will be used (in Fig. 1, coordinates are on the left, and the known optimal route with a length of 33551 is on the right).

If we number the rows of the original distance table starting from 0 , then the most distant cities will have numbers 3 and 16 . Route level 00 (Fig. 2 upper left) built from the initial two-member tour (by cities $3,16,3$ ) has a length of 34410 and a percentage ratio of $102.6 \%$ with the optimal route (hereinafter, percentages are indicated to the nearest tenth). The same figure shows the routes of level 01, level 02 (continuation of the first row of Fig. 2) and in the second row - the three longest routes of level 0 (which have the largest indices).

Sample construction 1. Route level 00 (Fig. 2 left upper) in one copy. It assumes the inclusion of the city with the number 30 in the two-member tour $(3,16,3)$ (other cities with similar embedding give a greater length of the resulting route of the zero level, and, accordingly, such routes will have a larger index. 


\section{N. Starostin et al. / Advances in Science, Technology and Engineering Systems Journal Vol. 4, No. 1, 268-281 (2019)}
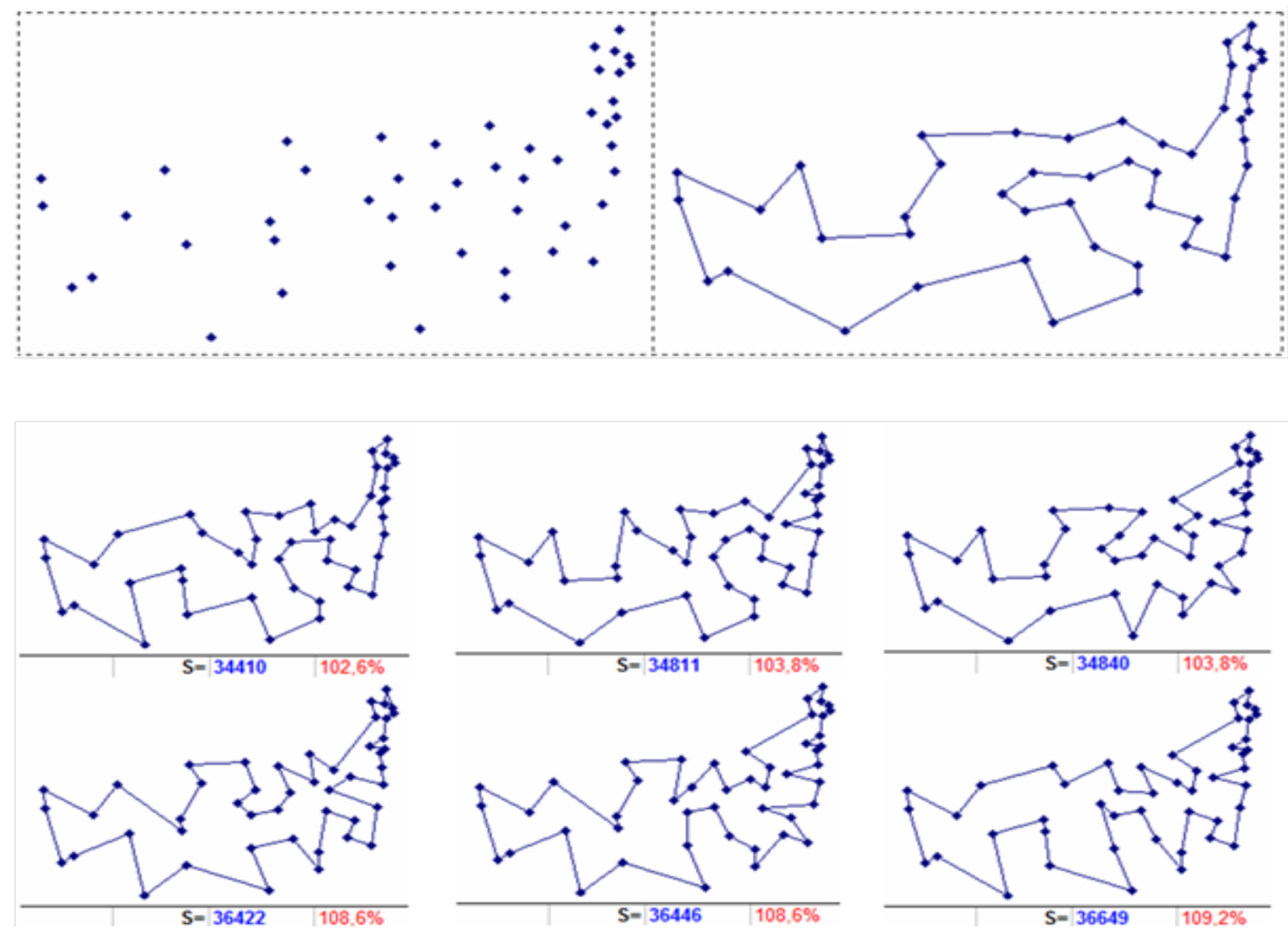

The route of level $10(00-10)$, built from the initial threemember tour $(3,30,16,3)$ is also one, its length is 33961 (Fig. 3 on the left). It implies embedding city 46 in the tour $(3,30,16,3)$ between cities 16 and 3 .

Level 20 routes (00-10-20) are already 15, their length has not improved, and has remained the same (33961) as at the previous (parent) level 10. Each of them involves embedding one of the 15 cities: $5,6,7,9,14,17,18,26,27,29,34,36,37,43,44$ into the initial tour in a certain place $(3,30,16,46,3)$. Next, for each newly formed tour routes of level $30(00-10-20$ - 30) are built, of which there are already about 152 , which also do not show the change in the length of the best tour. And only at level 40 there is a decrease in length in certain directions (Fig. 3 in the center and to the right).

First, we shall consider the direction corresponding to the shorter route length (33614 Fig. 3 on the right). Let us call it "branching 1". When building routes of level 50 from it, there will be several of them again, i.e., 22 , from each of which at level 60 will be somewhere along 23-27 similar new ones, and their length does not change (it remains equal to 33614). The situation is similar at the level of 70 and 80 . That is, there is a circumstance that "for a long time no changes are observed" of the lengths of routes with zero indices and therefore it is necessary to stop further constructions in this direction.

For the future, we should agree on the criteria for stopping calculations in the current direction. Let it be, for example, the following: "threefold repetition of the route length value with a zero index during the transition from level to level in the process of forming hereditary directions».
That is, in our case, calculations for this direction (branching 1) should be stopped after the formation of level 70 routes, despite the fact that we would reach the optimum (length of the route is $33551 \mathrm{~cm}$. Fig. 1) already while calculating from level 90, continuing calculations for this direction. For example, when calculating from the initial closed tour $3,1,33,7,30,16,32,19$, $46,31,4,3$ (when you include city 40 between cities 33 and 7). Or from another source tour $3,1,33,40,7,30,16,32,46,31,4,3$ (with the inclusion of the city 19 between cities 32 and 46 ).

Let us now consider the second direction ("branching 2"), corresponding to the greater length of the route (33741 Fig. 3 in the center). When building from it, 4 routes of level 50 are obtained with a length that has changed to a value of 33614. From each of the corresponding four routes, the eight-member initial tours are obtained at level of 60, with about 21-24 new routes in them, while their length does not change (it remains equal to 33614). At level 70 , another average of 23 routes is added. This is about $4 * 232$ new calculation directions in grand total. And if during the formation of the level 80 routes there is no change in the length of at least one of them, then, according to the entered criterion, the calculations in this direction ("branching 2") should be stopped, and therefore, in general, the entire "parent" direction of level 00 should cease. However, in reality this does not happen. When forming routes of level 80 from the initial closed tour 3, 40, 2, 8, $7,30,6,16,46,3$ (when you include city 32 between cities 16 and 46 - constructing at level 70), a route of length 33551 (Fig 1) results, which is an optimal one. 

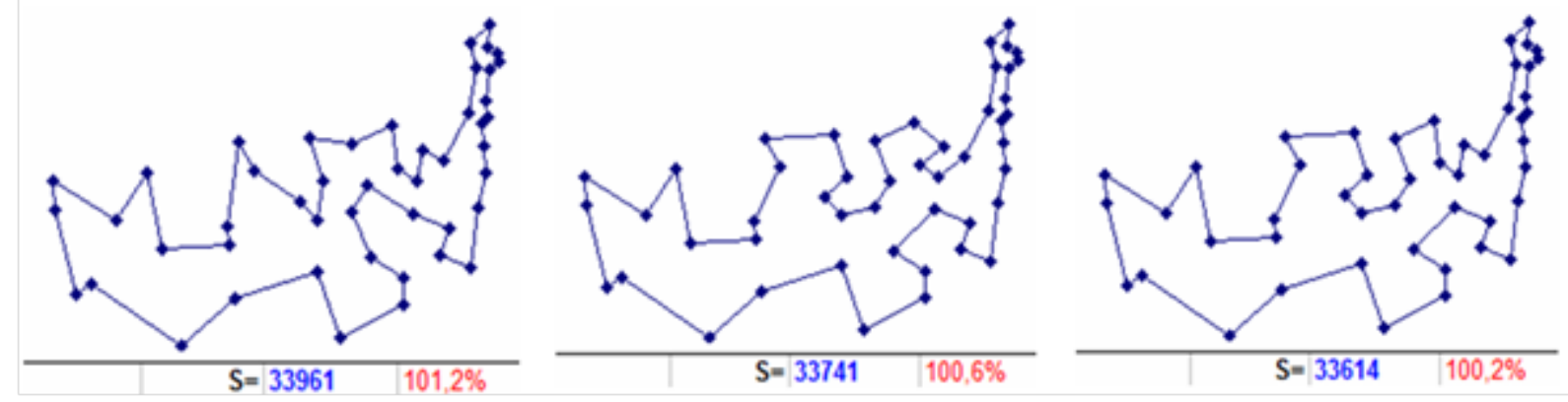

Figure 3. Route of level 10 (left), and of level 40 (center and right). Additionally shown are the length and deviation relative to the optimum.
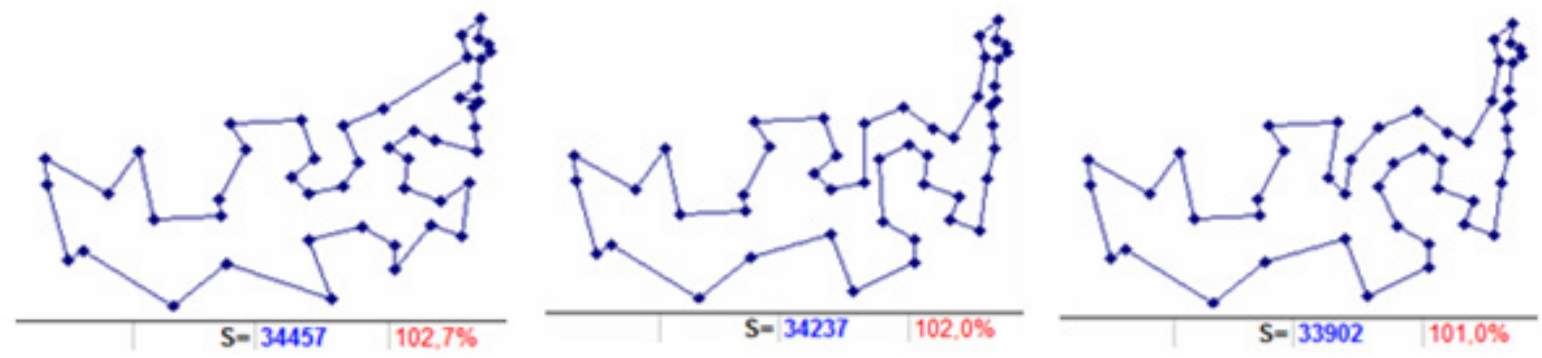

Despite the fact that the result within the declared scheme (1) has been achieved, we will continue our constructions from the $0 \mathrm{i}$ $(i \neq 0)$ levels in order to identify less labor-intensive directions. And such options are, for example, the following ones.

. This route is one copy. It assumes the inclusion in the two-member tour $(3,16,3)$ of the city with the number 32 . There are three routes of level $1_{0}\left(0_{24}-1_{0}\right)$, constructed from the original three-member tour $(3,32,16,3)$, their length is 34457 (Fig. 4 left). They suggest embedding in the tour (3, 32, 16, 3 ) cities 10,19 or 34 between cities 16 and 3 .

We will begin further constructions from city 10 (having a smaller sequence number). When calculating from the tour $(3,32$, $16,10,3$ ), the only route of level 20 is 34237 long (Fig. 4 in the center). It assumes embedding in the tour $(3,32,16,10,3)$ city 43 between cities 16 and 10. By performing this operation, we also get a single route of level 30 with a length of 33902 (Fig. 4 on the right). This, in turn, is assuming that the only embedding in the tour $(3,32,16,43,10,3)$ shall be city 22 between cities 3 and 10 . This embedding, in turn, results in obtaining an optimal route of length 33551 (Fig. 1). As a result, the entire calculation is significantly less time consuming than the calculations from level 00 in sample construction 1 . The remaining two branches (those from cities 19 and 34) are more computationally expensive.

Here we shall note that it is possible to dramatically increase the number of ways to build optimal routes through the use of extended schemes for non-deteriorating routes.

In [8] it was shown that within the framework of the scheme (1), as a rule, there are quite a few options for constructing optimal routes; however, in some cases it is not possible to quickly reach the optimal result, therefore in [7], alternatively, extended schemes are proposed. They allow, for example, in problems with a dimension of up to 50 elements, to obtain an optimum often even at the initial state. Either they open new directions in which the optimal result is achieved in the absence of significant branching of the process, or at earlier than in scheme levels (1), which may already be more relevant for tasks in the range of dimensions, from 50 to 100 elements [9].

The first version of the extended calculation scheme:

$$
\begin{aligned}
& \text { level }(0-1)_{i}-\text { level } 2_{0}-\text { level } 3_{0} \ldots \\
& (i=0,1,2, \ldots)
\end{aligned}
$$

Here, level $(0-1)$ represents some combination of level 0 and level 1 from the previous scheme. Full routes (Hamiltonian cycles) from level 0 are not built, and each of the $n-2$ source 3-member tours i1, ik, i2, i1, $(\mathrm{k}=3,4, \ldots, \mathrm{n})$ is converted into 3 four-member one, from which the full routes are already formed from the combined level (0-1). Here, they are presented in the number of (n $2) *($ n 3$) * 3$, which is a broader basis for the formation of full routes of levels 20 and subsequent levels. At the same time, within the framework of the scheme (1) for the formation of level 20 routes, there is, as practice shows, in all directions (n 2) or several more closed source routes, that is, a small subset of the analog from the scheme (2).

Let us explain the scheme (2) in our current example. Among the many variants of the initial routes corresponding to the level $(0$ - 1) i, we take two (with some average level indicators of the full route length) and at the same time non-existent (unrealizable) within the framework of the scheme (1).

Sample of construction 3. Let the first of them be the original route that implies embedding into a two-member tour 3, 16, 3 pairs of cities 15 and 30 between cities 3 and 16 . As a result, we have tour $3,15,30,16,3$, and the only route from it is on level 20 , involving the incorporation of city 37 between cities 30 and 15, with a total length 34410, corresponding to the upper left in Fig. 2 

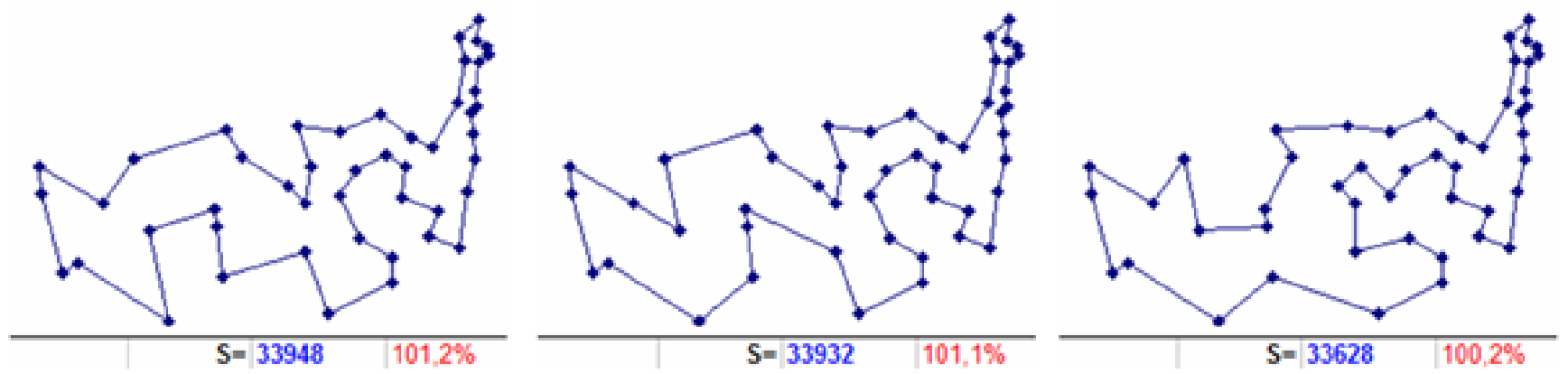

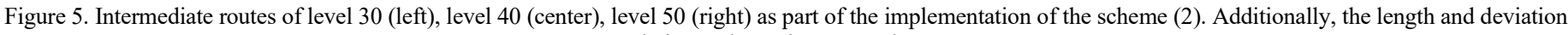
relative to the optimum are shown.

In turn, it builds only one next-level route with a zero index (level 30), with an estimated integration of city 10 between cities 15 and 37 on tour $3,15,37,30,16,3$ with the result (length 33948) indicated in Fig. 5 left. Already from this position is also obtained the only route of level 40 with the expected inclusion of city 25 between cities 3 and 15 in tour $3,15,10,37,30,16,3$, and with length of 33932 (Fig. 5 in the center). Further, the route of level 50 is also created in a single copy indicating the inclusion of city 12 between cities 15 and 10 in tour 3, 25, 15, 10, 37, 30, 16, 3, and with length of route 33628 (Fig. 5 on the right). And already from that, after the implementation of the specified inclusion, we immediately get the optimal route (Fig. 1 on the right). As a result, all transitions from the initial to the last level pass without a single branching in the process of construction.

Sample of construction 4. Let us take, as the second similar initial tour, a route with a supposed embedding in the two-member tour $3,16,3$ pairs of cities 22 and 30 between cities 3 and 16 . There is also no corresponding route within scheme (1), since embedding from level 1 with city 22 in the case of constructions from level 00 (or with city 30 in case of constructions from level 025) will have an index number of level 1 greater than 0 , which is beyond the scope of the scheme. Within the framework of scheme (2), the corresponding full routes exist and coincide, their length is 35935 (Fig. 6, left). The calculation from tour 3, 22, 30, 16, 3 gives a single route of level 20 , which implies embedding city 11 between cities 22 and 30 with a full route 34694, corresponding to the central route in Fig. 6. There are already two level 30 routes (Fig. 6 on the right), they suggest embedding cities 32 or 45 between cities 3 and 16 in tour $3,22,11,30,16,3$. Both routes with the specified embedding lead to an optimal result (Fig. 1 right).

Sample of construction 5. It is also possible to note the possibility of obtaining an optimum according to the scheme (2) among the directions existing within the framework of the scheme (1), but "rejected" by the criterion of stopping the calculations. For example, in the framework of scheme (1), according to this criterion, constructions are rejected from the direction (06-10-2030 ) because of the fourfold construction at each level of the same complete route. Namely, the sequential inclusion in the twomember tour $3,16,3$ the city $43(3,43,16,3)$, then the city $10(3$, $10,43,16,3)$. After that, city $40(3,40,10,43,16,3)$ and finally, city $0(3,40,10,0,43,16,3)$, each time they build the same full length route $33633(100,2 \%$ of the optimum of Fig. 7, in the center). In fact, there is a threefold repetition of the length of the route and, according to the criterion, the calculation stops.

Let us present another extended scheme similar to (2), but with three levels combined.

$$
\text { level }(0-1-2)_{i}-\text { level } 30-\text { level } 40 \ldots
$$$$
(i=0,1,2, \ldots)
$$

It provides even greater opportunities for obtaining recordbreaking results due to the impressive "re-selection" component of the combined entry level. $(0-1-2)$.

Sample of construction 6. So, for example, thanks to such a scheme, from tour $3,12,10,43,16,3$ (which corresponds to the construction from the combined level $(0-1-2))$ in our current example of 48 cities, we immediately get the optimal route. This tour is not the only one on the level combined by the scheme (3).

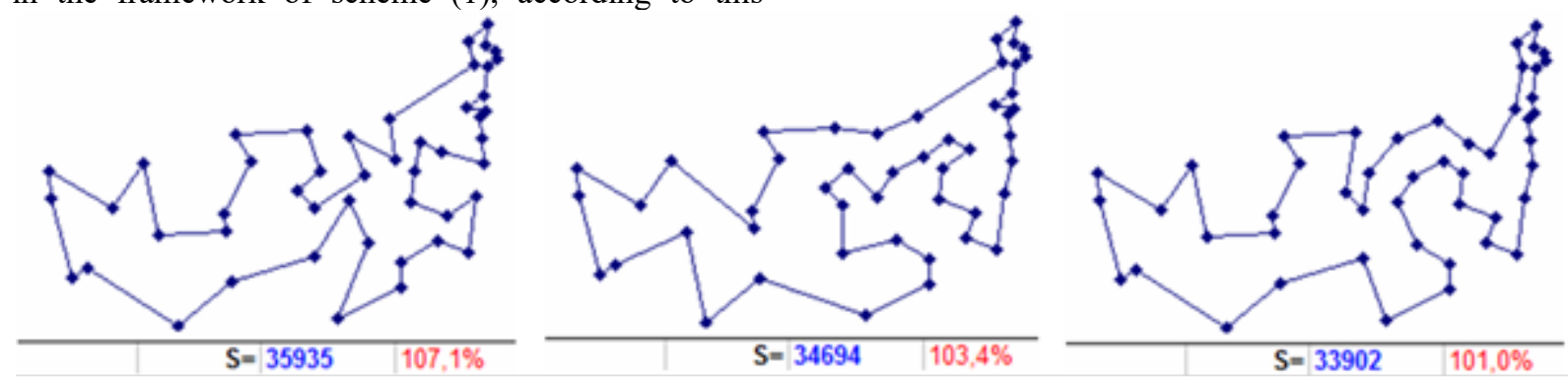

Figure 6. Intermediate routes of the "combined" level (0-1) i (left), level 20 (center), level 30 (right) as part of the implementation of the scheme (2). Additionally, the length and deviation relative to the optimum are shown. 


\subsection{Calculation based of the scheme with permissible deterioration of routes}

Such a scheme is designed to provide flexibility in achieving record routes. This is a way to improve from a slightly worse calculation position (which is longer than the best route of the current level), which at the next or later levels can lead to a change in the current minimum in the considered direction of calculation. It is significant that this allows to avoid the situation "for a long time, no deviations from the found tour length are observed". This approach is implemented based on the use of index variation not only as previously at the zero level, but also at subsequent levels of construction. That is, in the general case, the calculation scheme can be as follows [16]:

(2) level $0_{i 0}-$ level $1_{i 1}-$ level $2_{i 2} \ldots(i 0, i 1, i 2, \ldots=0,1,2, \ldots)$

The total number of calculations here is much larger relative to the scheme (1). Therefore, from a practical point of view, one should artificially limit the potential excessive growth of the levelby-level calculations. And from this position, it is more expedient to introduce into (4) additional restrictions or to use some hybrid variants from schemes (1) - (3) and (4), for example let us consider the scheme (5), which is defined by the next three paragraphs.

If at the levels of $\mathrm{J} 0$, where $\mathrm{J}=1,2,3 \ldots$, only one route is observed (with index 0 ), then further constructions lead only from the initial $(\mathrm{J}+2)$-member tour for it, i.e., according to scheme (1).

If there are several routes with index 0 , then further constructions will lead not only from the initial $(\mathrm{J}+2)$-member tours for them, but also from all other $(\mathrm{J}+2)$-member tours that are initial for the level routes with corresponding non-zero indices, i.e., as in scheme (4).

Moreover, in order to reduce computations, directions of calculations from non-zero indices, for constructions from which the full route with a zero index of the next level coincides with the parent route (that is, in the absence of a decrease in the length), shall not be considered,).

To illustrate the scheme with permissible degradations of routes, we turn to the already considered version of the calculation from construction sample 1 , where for the first time instead of one, in the direction of 20 (in the direction $00-10-20$ ), 15 routes appear with a zero index and not improved length of 33961 (Fig. 3 left). This is just the reason to use the scheme (5).

Sample of construction 7. So, we shall start constructing routes of the 3rd level. Embedding in a certain place of the initial tour (3,
$30,16,46,3$ ) each of the identified cities corresponding to the level 2 zero index does not change the generated routes of the next level with the zero index (see construction pattern 1, level 30). According to the adopted scheme, we will construct from non-zero level indices. So embedding of city 15 between cities 3 and 30 (corresponding to the route with the last, i.e., the largest index of level 2 with an initial length of 35582) results in two routes of level 30 with a decrease in length to 33614 .

Further, from the first of these routes, through the implementation of the supposed embedding of city 33 between cities 3 and 15 (on tour $3,15,30,16,46,3$ ), 25 routes of level 40 are obtained. In one of them, the embedding of city 32 between cities 16 and 46 (in tour 3, 33, 15, 30, 16, 46, 3) allows to obtain the optimal route which is several levels earlier than indicated in construction sample 1.

From the second route, an optimal tour is also obtained, but it is built one level further.

Sample of construction 8. A stronger result is obtained when constructing from level 06, where there is (before the start of the intended embedding of city 43 in the two-member tour 3, 16, 3 ) a full route (Fig. 7, left) 35143 in length. The route of level 10 from the implementation of such embedding is one, with length of 33633 (Fig. 7, in the center) and it involves embedding the city 10 between 3 and 43 (on tour 3, 43, 16, 3). When implementing it, we already get 10 level 20 routes and, according to scheme (5), along with them, it is possible to conduct calculations also from levels $2 \mathrm{i}$ (where $i \neq 0$ ). As a result of the calculation from level 27 (where the full route 34479 in Fig. 7 is on the right), when the city 22 is embedded between 3 and 10 (in tour 3, 10, 43, 16, 3), we immediately get the optimal route (Fig. 1 on the right).

It can be noted that in a similar way and with the same laboriousness (when building from level 2), optimum is obtained when calculating from the starting level 012 (the full potential route is no longer than 35808), with an estimated only integration of city 10 into the two-member tour $3,16,3$.

\section{Natural innovation in the basic calculation procedure}

Having considered the main directions of calculations, and based on the statistics of the calculations carried out earlier [7-9, 16], we can draw some conclusions. For the considered possible directions of calculations in various test examples with dimensions up to 100 and a little more elements, it is always possible to obtain the optimal result with varying degrees of complexity. In this case, the optimum for each of the possible calculation schemes, as a rule, is achieved in many different ways. The least laborious route
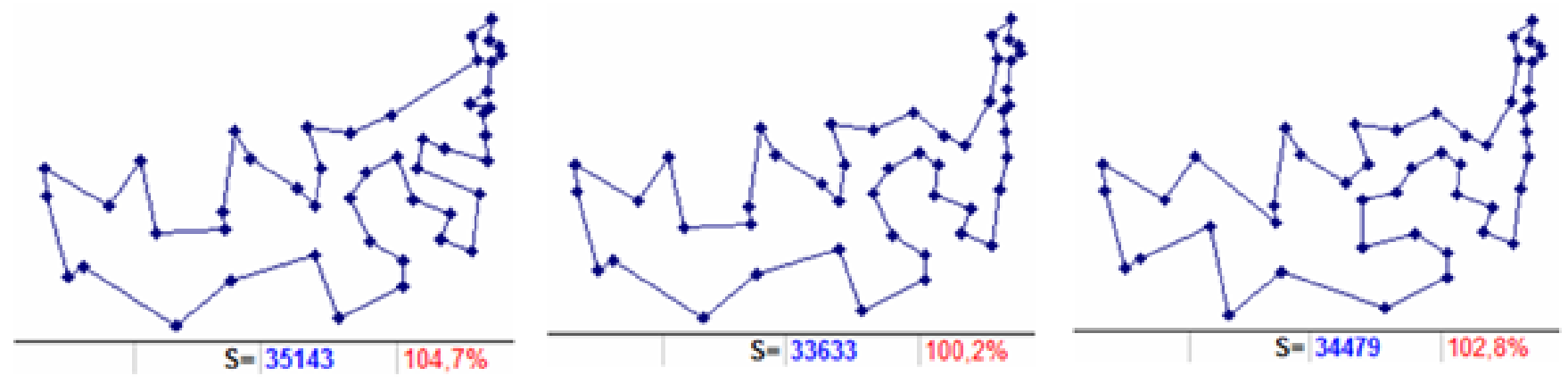

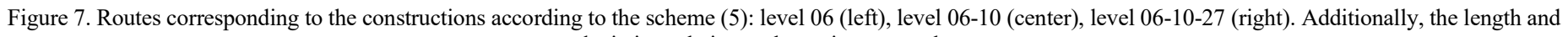
deviation relative to the optimum are shown. 
(when comparing calculations from level 0 starting tours) was often the one where the optimal route is built at earlier levels. Less often - at later levels, but in the absence of a large number of branches. The results of experiments [7-9, 16] also show the following. The more extensive the base of the starting (initial) level, as is the case with the use of advanced schemes, the higher the probability of an "earlier" approach to optimum in individual calculations from starting tours. The total number of such exits is also growing.

There is a natural opportunity to significantly increase the number of zero level starting tours from the current $n-2$ options within the framework of the scheme (1). That will also increase the number of corresponding approximate level routes. The essence of such an extension is as follows. For convenience and certainty, the baseline calculation procedure used the directive choice of the first two cities (maximum distance from each other). From this pair, all subsequent constructions were carried out. If calculations would be carried out from each unique pair of source cities, then the number of starting two-member tours increases to $n *(n-1) / 2$, where $n$ is the number of cities. Let us return to current example (48 cities) to clarify application of new starting conditions.

Samples of construction 9. In practice, probably, such a large number (in our new conditions 1128) of initial tours is not necessary. Let us see what happens if we restrict ourselves, for example, to considering only a few starting two-member tours, from which when forming routes of the zero level we get the smallest routes with zero index (i.e., the smallest among all possible routes of levels 00 ).

In our example, there are only 68 (out of 1128) two-member tours with a minimum route (at level 00) of a length of 33614 (as in Fig. 3 on the right). By constructing according to scheme (1), we will reveal among them those two-member tours, from which the optimal route will be constructed at the nearest of the subsequent levels, and accordingly the number of this level. Thus, directly from all possible three-member tours, being built from each of the 68 two-member tours, the optimal route is not immediately reached. But for the individual subsequent fourmember tours the optimal route (Fig. 1 on the right) is already calculated. That corresponds to the constructions from level 10. The code of these constructions, corresponding to scheme (1), is contained in table 1; it indicates the sequence of obtaining the optimal route (length 33551 ).

Table 2 contains similar information, but this time from a small sample of the remaining starting two-member tours. In it, the best routes of zero levels (routes of level 00) exceed the minimum length of 33614 (that is, more than the tours from the previous table). The corresponding constructions for each of those twomember tours of scheme (1) also lead to the identification of the optimal route, with the implementation of inclusion from level 10.

The above tabular data suggests that there are no clear preferences when choosing a two-element tour from the available starting conditions in order to obtain a "short" calculation of the optimal route. For example, our initial starting two-member tour (3 163 ) from the most distant cities is with index 75 according to the criterion for the route length of level 00 almost exactly in the middle of the totality of 1,128 two-member tours (among the sequence numbers in ascending order from 532 to 574). From the point of view of building an optimal route (sample of building 1 8 ), it is not very fast relative to others. But the starting two-member tour 123812 with the sequence number 1127 (Table 2 last line) with the length of the 00 level route equal to 35400 (Fig. 8 on the left) is almost perfect from the same point of view (like some others presented in both Tables).

And this is despite the fact that its starting indicators are next to the last ones in proximity to the optimum length of its best route among all 1128 results of levels 00 . However, the optimal path is quickly and unequivocally (without a single branching) built from level 03 (basic length 35659 in Fig. 8 in the center). Specifically, first begins the inclusion in the two-member tour of the city 28 (12 2838 12). As a result, a route with the length of 34603 is built (Fig. 8 on the right). Then, the only possible inclusion of the next level 10 is the insertion of city 32 (12 322838 12) and, as a result, the already known optimal route is obtained (Fig. 1 on the right).

Table 1: Sequential Data

\begin{tabular}{|c|c|c|c|c|c|}
\hline $\begin{array}{l}\text { The sequence } \\
\text { number of the } \\
\text { original tour }\end{array}$ & $\begin{array}{l}\text { The order of the } \\
\text { cities for the } \\
\text { starting two- } \\
\text { member tour }\end{array}$ & $\begin{array}{l}\text { The length of the level } 0 \mathrm{i} \\
\text { tour(at the beginning of the } \\
\text { direction for constructing } \\
\text { the optimal route) }\end{array}$ & $\begin{array}{l}\text { Order of cities } \\
\text { of a three- } \\
\text { member tour } \\
\left.\text { (level } 0_{i}\right)\end{array}$ & $\begin{array}{c}\text { Length of } \\
\text { a tour of } \\
\text { level } 1_{0}\end{array}$ & $\begin{array}{l}\text { Order of cities in a } \\
\text { four-member tour, } \\
\text { directly from which the } \\
\text { optimal route is built } \\
\left(\text { level } 1_{0}\right)\end{array}$ \\
\hline 3 & 1301 & $\begin{array}{l}35374 \\
34110 \\
\end{array}$ & $\begin{array}{llll}1 & 12 & 30 & 1 \\
1 & 15 & 30 & 1 \\
\end{array}$ & $\begin{array}{l}33628 \\
33628 \\
\end{array}$ & $\begin{array}{l}11512301 \\
\\
\end{array}$ \\
\hline 8 & 1431 & $\begin{array}{l}35137 \\
35131 \\
\end{array}$ & $\begin{array}{llll}1 & 22 & 43 & 1 \\
1 & 24 & 43 & 1 \\
\end{array}$ & $\begin{array}{l}33879 \\
33879 \\
\end{array}$ & $\begin{array}{c}12224431 \\
\quad / / \\
\end{array}$ \\
\hline 22 & 6376 & $\begin{array}{l}34947 \\
35613 \\
\end{array}$ & $\begin{array}{llll}6 & 22 & 37 & 6 \\
6 & 24 & 37 & 6 \\
\end{array}$ & $\begin{array}{l}33955 \\
33955\end{array}$ & $\begin{array}{c}62224376 \\
\quad / / \square\end{array}$ \\
\hline 39 & 173717 & 35191 & 17243717 & 33955 & 1722243717 \\
\hline 45 & 222722 & 34483 & 22242722 & 33955 & 2224372722 \\
\hline 53 & 273727 & $\begin{array}{l}34704 \\
35769 \\
\end{array}$ & $\begin{array}{llll}27 & \mathbf{1 2} & 37 & 27 \\
27 & \mathbf{2 4} & 37 & 27 \\
\end{array}$ & $\begin{array}{l}34701 \\
33955 \\
\end{array}$ & $\begin{array}{lllll}27 & \mathbf{2 8} & 12 & 37 & 27 \\
27 & \mathbf{2 2} & 24 & 37 & 27 \\
\end{array}$ \\
\hline
\end{tabular}


Table 2: Tour Measurements

\begin{tabular}{|c|c|c|c|c|c|c|}
\hline $\begin{array}{r}\text { Tour } \\
\text { Number }\end{array}$ & $\begin{array}{l}\text { Order of starting } \\
\text { cities according to } \\
\text { the scheme (1) tour }\end{array}$ & $\begin{array}{l}\text { Index among } \\
\text { all tours of } \\
\text { levels } 0_{0}\end{array}$ & $\begin{array}{l}\text { Tour length } \\
\text { of level } \theta_{0}\end{array}$ & $\begin{array}{l}\text { Tour length of } \\
\text { level } 0 \mathrm{i} \text { (starting } \\
\text { to calculate) }\end{array}$ & $\begin{array}{l}\text { Order of three- } \\
\text { member tour cities } \\
\left(\text { level } O_{i}\right)\end{array}$ & $\begin{array}{l}\text { Tour length of } \\
\text { level } 1_{0}\end{array}$ \\
\hline 69 & 3103 & 1 & 33633 & 35877 & 322103 & 34479 \\
\hline 70 & 3433 & 1 & 33633 & 35254 & 322433 & 34479 \\
\hline 331 & 243824 & 33 & 34151 & $\begin{array}{l}35539 \\
36239 \\
\end{array}$ & $\begin{array}{llll}24 & 21 & 38 & 24 \\
24 & 40 & 38 & 24 \\
\end{array}$ & $\begin{array}{l}34668 \\
34151 \\
\end{array}$ \\
\hline 332 & 244324 & 33 & 34151 & $\begin{array}{l}35131 \\
35420 \\
36138 \\
35880\end{array}$ & $\begin{array}{llll}24 & 1 & 43 & 24 \\
24 & 8 & 43 & 24 \\
24 & 22 & 43 & 24 \\
24 & \mathbf{4 0} & 43 & 24\end{array}$ & $\begin{array}{l}33879 \\
33985 \\
33879 \\
34151\end{array}$ \\
\hline 333 & 384338 & 33 & 34151 & 36575 & 38224338 & 34510 \\
\hline 394 & 222422 & 46 & 34276 & $\begin{array}{l}35881 \\
35128 \\
35561 \\
36138 \\
34483 \\
36885 \\
36138\end{array}$ & $\begin{array}{llll}22 & \mathbf{1} & 24 & 22 \\
22 & \mathbf{6} & 24 & 22 \\
22 & \mathbf{8} & 24 & 22 \\
22 & \mathbf{1 7} & 24 & 22 \\
22 & \mathbf{2 7} & 24 & 22 \\
22 & \mathbf{3 7} & 24 & 22 \\
22 & \mathbf{4 3} & 24 & 22\end{array}$ & $\begin{array}{l}33879 \\
33955 \\
33985 \\
33955 \\
33955 \\
33955 \\
33879\end{array}$ \\
\hline 639 & 224322 & 90 & 34479 & $\begin{array}{l}36427 \\
35137 \\
35254 \\
35818 \\
36152 \\
36138 \\
37297\end{array}$ & $\begin{array}{llll}22 & \mathbf{0} & 43 & 22 \\
22 & \mathbf{1} & 43 & 22 \\
22 & \mathbf{3} & 43 & 22 \\
22 & \mathbf{9} & 43 & 22 \\
22 & \mathbf{2 0} & 43 & 22 \\
22 & \mathbf{2 4} & 43 & 22 \\
22 & \mathbf{3 8} & 43 & 22\end{array}$ & $\begin{array}{l}33991 \\
33879 \\
34479 \\
33991 \\
34094 \\
33879 \\
34510\end{array}$ \\
\hline 854 & 102210 & 156 & 34755 & $\begin{array}{l}35877 \\
35022\end{array}$ & $\begin{array}{llll}10 & 322 & 10 \\
10 & \mathbf{2 8} & 22 & 10\end{array}$ & $\begin{array}{l}34479 \\
34094\end{array}$ \\
\hline 1127 & 123812 & 258 & 35400 & 35659 & 12283812 & 34603 \\
\hline
\end{tabular}
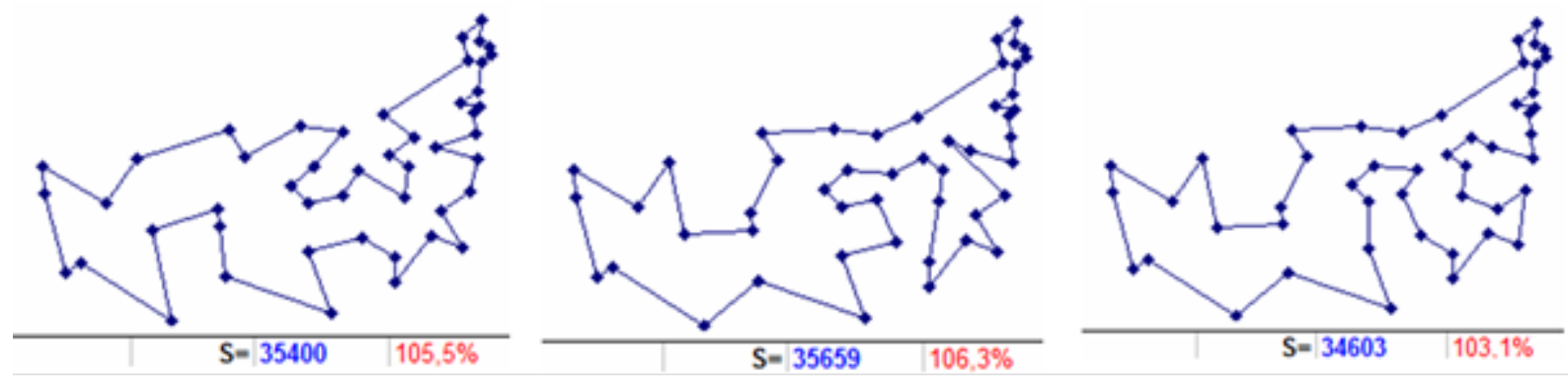

Figure 8. Routes from the starting cities 12 and 38 corresponding to the constructions according to the scheme (1): level 00 (left), level 03 (center), level 03 - 10 (right). Additionally, the length and deviation relative to the optimum are shown.
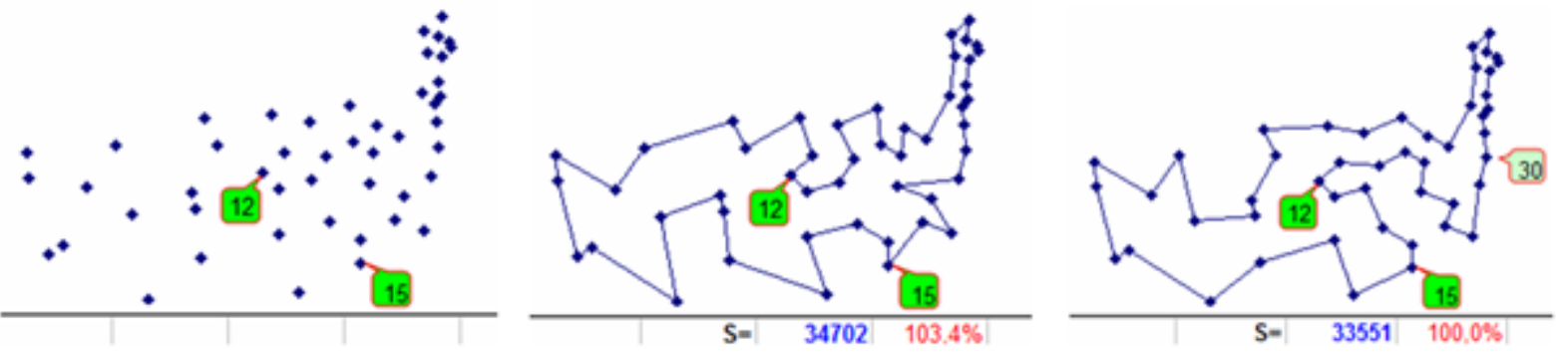

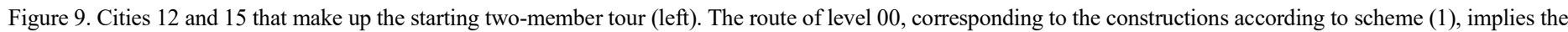

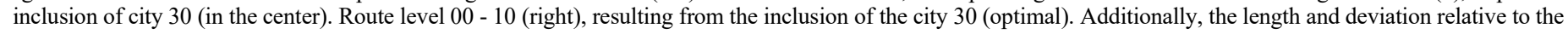
optimum are shown. 

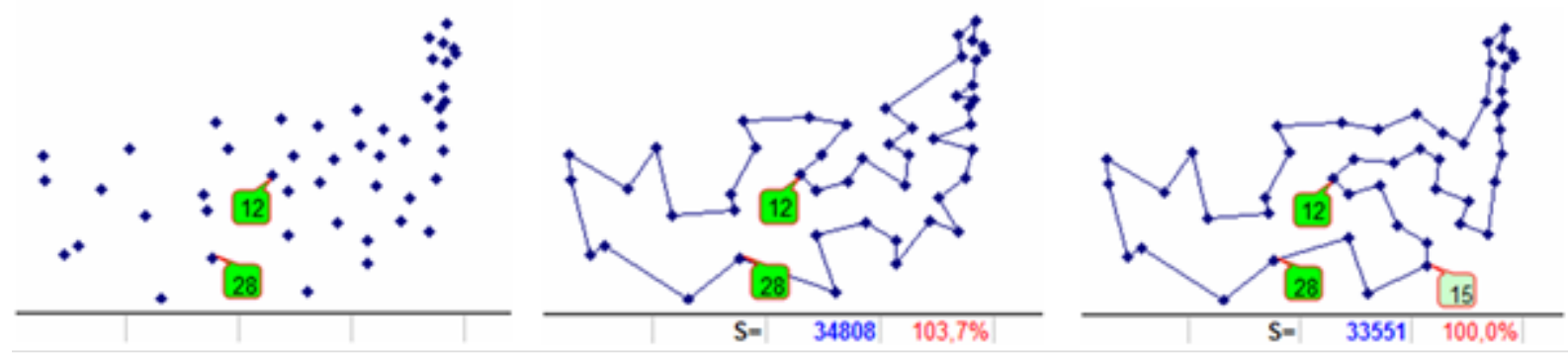

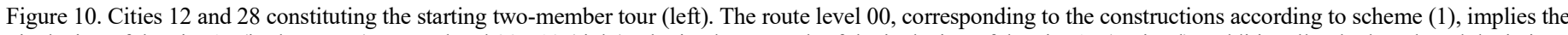

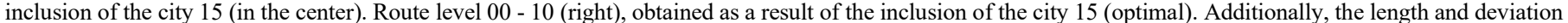
relative to the optimum are shown.

Samples of construction 10. It is necessary to separately distinguish the group of constructions where the optimal tour is obtained at the earliest stage (from the beginning of the calculations) when implementing the inclusion in the starting twomember tour of the third city, i.e., after calculations at the zero level.

Fig. 9 (left) shows an illustration of the starting conditions for the calculation of a two-member tour between cities 12 and 15 . In the center of this figure, the shortest route (length 34702) is being built for those cities which have a zero level route (level 00). It is the only one and involves the inclusion in the two-member tour of the city 30 . After the realization of this inclusion, we obtain the optimal route Fig. 9 (right).

Also, when implementing the inclusion of level 00, an optimal route is constructed from a two-member tour between cities 12 and 28. Fig. 10 (left) is an illustration of the starting calculation conditions for this two-member tour. In the center of the figure, the shortest (length 34808) zero level route (level 00) is shown. There are two such routes and, accordingly, two directions of construction according to the scheme (1). The first one, which implies the inclusion of city 2 , is a dead end one for reaching the optimum. The second one, which implies the inclusion of the city 15 , after its implementation immediately builds the optimal route Fig. 10 (right).
For more commonality, Fig. 11 shows 4 more routes of the zero level from binomial tours, but with a non-zero index (levels $0 \mathrm{i}, \mathrm{i}$ $\neq 0$ : the first row in the center and to the right, the second row in the center and to the left). Each of the directions corresponding to them, after the implementation of the proper inclusion of the third city in the corresponding route of the two-member tour, also immediately leads to an optimal result (Fig. 11, second row rightmost). This happens again when constructing from the zero level, but only already when implementing the inclusion corresponding to a non-zero index (from the level $0 \mathrm{i}, \mathrm{i} \neq 0$ ).

Unfortunately, in the absence of a general criterion for choosing the "correct" pairs of starting two-member tours, in order to identify all three-member tours, from which the optimal route is immediately constructed, 17296 possible options will have to be considered (in general, $\mathrm{n} *(\mathrm{n}-1) *(\mathrm{n}-2) / 6$, where $\mathrm{n}$ is the dimension of the problem).

\section{Features of finding the best route in tasks with a large dimension}

In the light of the innovations outlined in the previous section, at the very beginning, there emerges a very large number of directions of calculations from each of which it is potentially possible to get the best route.

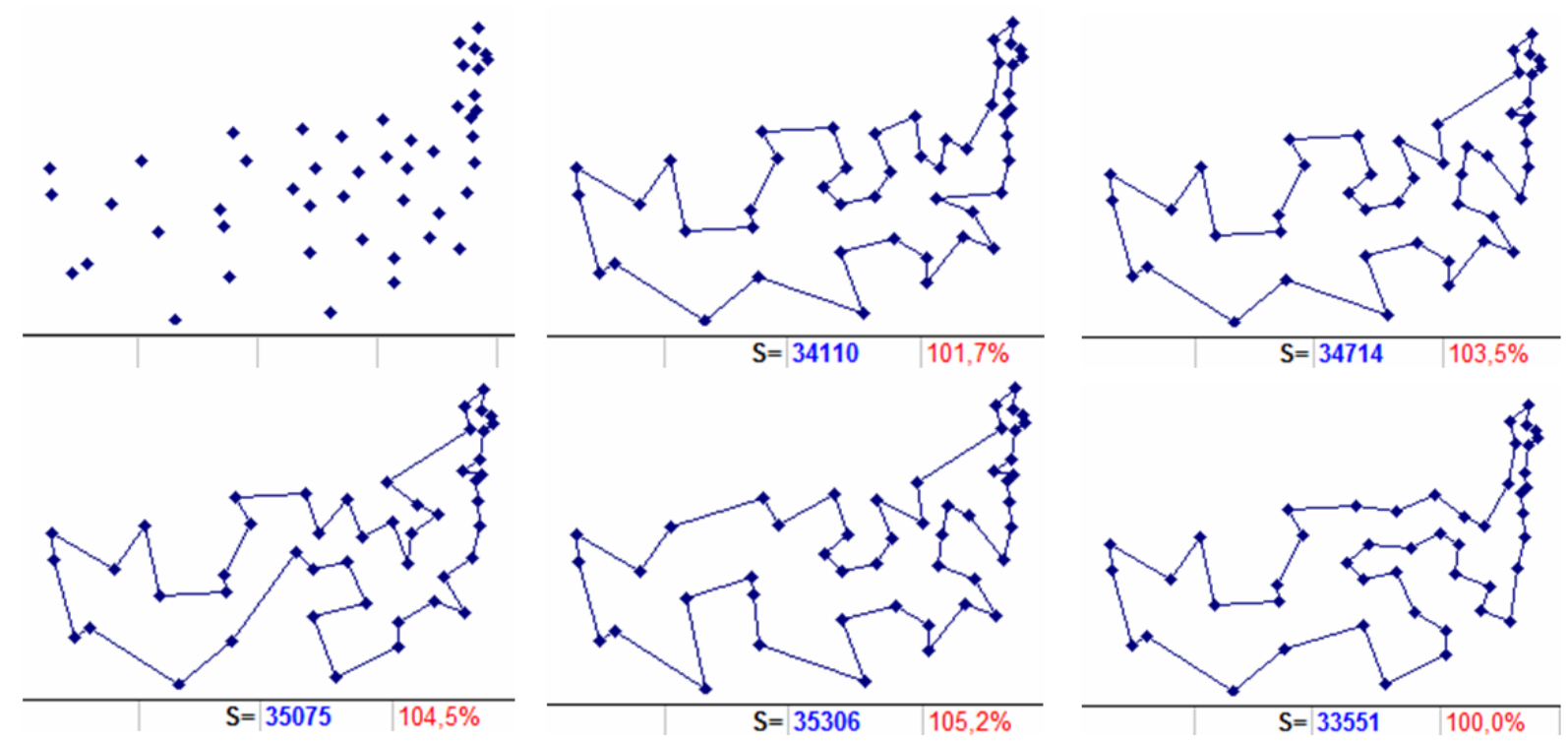

Figure 11. The mutual position of 48 cities (upper left). The optimal route is 33551 long (bottom right). The rest: routes of level 0i, directly transformed after the inclusion of the corresponding third city in the optimal route. Additionally, the length and deviation relative to the optimum are shown. 
In such examples as ours (dimensionality of 48 cities) and even twice as large dimensionality, it is still possible to authorize the definition of not only the best route, but also the youngest level where it is reached (along with a full set of options for building it on it). This was shown in construction samples 10 .

For examples with a dimension somewhat greater than 100 elements, such an approach (i.e., the calculation of "breadth") is already quite laborious. Therefore, we first denote a simplified version of the organization of calculations among the set of initial two-member tours. For each unique pair of source cities, determine the minimum route of level 00 , and the corresponding city for inclusion in the two-member tour (if there are several, then one of them). Index all ( $n *(n-1) / 2)$ obtained routes in order of increasing length (we call these indices global). Limit the number of calculations from starting two-member tours (or groups of tours) only to those of them that have small global indices.

In addition, we will somewhat limit the number of calculations in scheme (5). One of its possible modifications can be formulated as the scheme (6) determined by the next paragraph.

If at the levels of $\mathrm{J} 0$, where $\mathrm{J}=1,2,3 \ldots$, only one route is observed (with index 0 ), then further constructions will only lead from the initial $(\mathrm{J}+2)$-member tour, i.e., according to the scheme (1).If there are several routes with index 0 , then further constructions will lead not only from the initial $(J+2)$-member tours for them, but also from all other $(\mathrm{J}+2)$-member tours that are initial for the level routes with corresponding non-zero indices, i.e., as in the scheme (4). At the same time, in order to further reduce the computations, as compared with scheme (5), we shall consider only separate directions of calculations from non-zero indices of the current level ("current-baseline"). These are the directions from which a chain of routes with zero indices of subsequent levels with decreasing lengths is built without branching. Moreover, the length at the end of the chain (before branching occurs), even if only one link actually turns out, should be less (or at least not more) than the length of the route with zero index of the "current-base" level.
Let us consider how this is implemented using the example of calculation, for a test with a dimension of 131 elements (Fig. 12, top row to the left) from the ASIC data set of the VLSI TSP collection (file xqf131.tsp) [20]. For convenience, we will use the numbering of the test elements (in this case, vertices or points) defined by a set of Cartesian coordinates, starting from 0 (and not from 1 , as in the original source [20]).

Sample of construction 11 (from a two-member tour with the global index of 0). Among the routes of level 00, constructed from all possible combinations of initial two-member tours, the shortest length is 580.23 (Fig. 12 upper row in the center - for simplicity, we shall ignore digits after hundredths) and, accordingly, two of them have a global index of 0 . The first one combines the vertices 2866 and the second one has the vertices 2862 . Both assume that vertex 111 is included in the initial tour. Let us consider the constructions from the first one.

Thus, from the three-member tour (28 1116628$)$ a minimum route is built (Fig. 12 upper row to the right) with a length of 579.99. It predetermines 3 variants of the inclusion vertices $(25$, 26 or 18) corresponding to the zero level index, and therefore along with them, according to scheme (6), other remaining vertices of the possible inclusion should be considered. Among all the directions, the inclusion of the vertex 34 (28 341116628 ) seems to be one of the most promising for further development. After it, a diminishing chain of lengths for complete routes is constructed with zero indexes of subsequent levels up to level 5, where there are three routes with zero index length 574.77 (Fig. 12 bottom row to the left) again. Therefore, the condition of the scheme (6) for the final length of the chain is less than the length of the route with the zero index (579.99) of the "current-base" level. Since then there are three inclusions (from zero level indices) into a seven-member tour (28 34971111069666 28), all remaining vertices with nonzero indices should also be considered. At this stage, there is a good potential for the development of directions at the inclusion vertices with zero indices (60 94 and 95). Consider the first of them - the inclusion of vertex 60 (28 34 9711110696606628 ), from which a sequence of decreasing routes to level 8 is unambiguously constructed, where 36
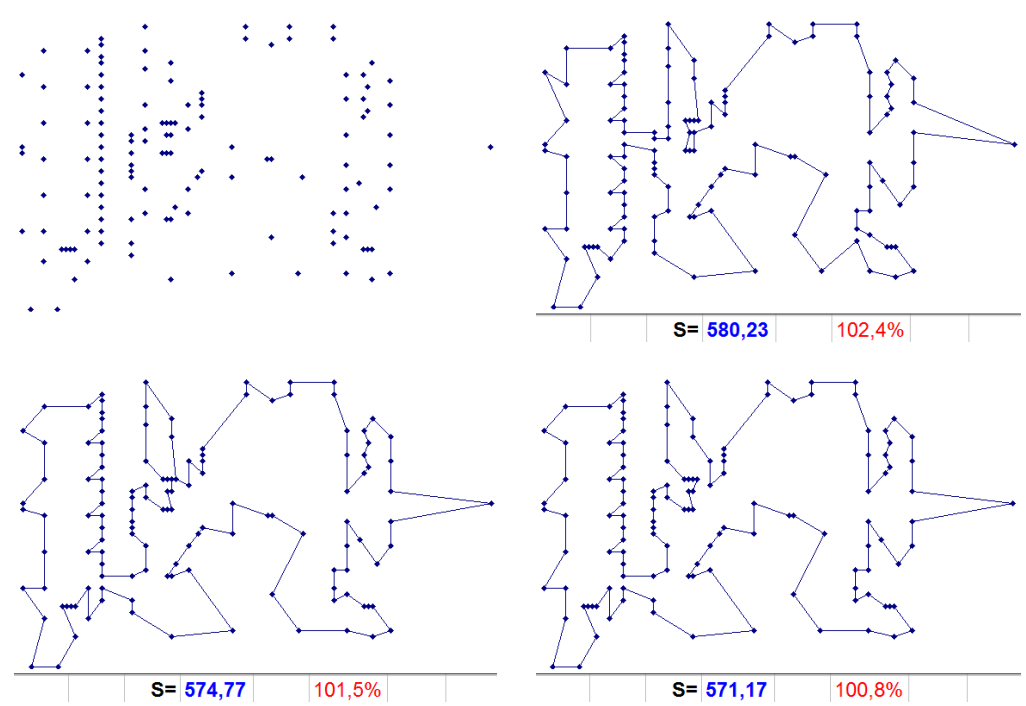
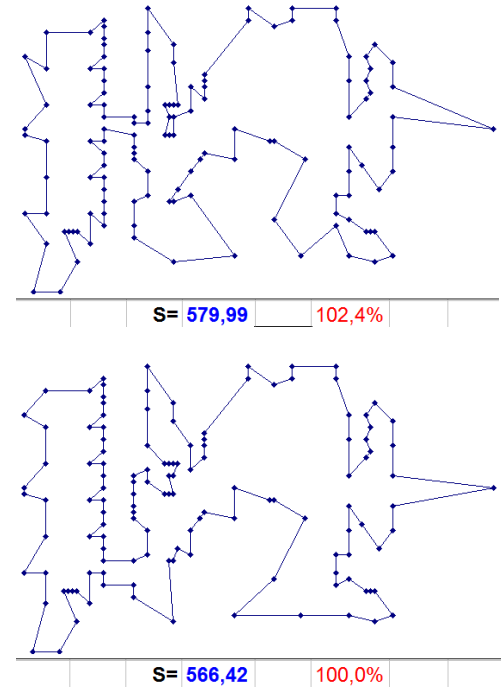

Figure 12. The mutual position of 131 points (top left). Record route length 566,42 (bottom right). The rest: a sequence of key intermediate routes. Additionally, the length and deviation relative to the record are shown. 
directions (vertices) characterizing routes with a length of 571.17 with a zero index appear (Fig. 12 bottom row in the center). Any of them does not reduce the length of the route with a zero index on the next level. But from one of them - from the inclusion of vertex 93 in the ten-member tour (28 341193971111067979 $606628), 44$ more routes are formed with a zero index and with the same length. And here already (when constructing routes of the next level), a record 566.42 length is not improved (Fig. 12 bottom row to the right), from the inclusion of vertices corresponding to non-zero level indices. This happens when vertices 77, 87 (correspondence to index 4) or 13 (correspondence to index 9) are included between vertices 1193 in tour 28341193971111069679606628 .

Here is the record tour: 666257585960727179788283 84858990949596103102110109108114118115119116 12112812712613012512412311210710610510110099 1041131171201291221119788929893918786818077 76746763735244252618241615141317124115067 12893102343424140392238373621353433203231 301929282745535446474849505156556164686569 757066 .

It is achieved according to the scheme (6), at constructions from level $9(00-163-20-30-40-50-60-70-80-9 \mathrm{i}$, где $\mathrm{i}=4,9)$, which is two levels earlier than at calculation according to scheme (5) from a two-member tour with the most distant cities in [15]. If the optimal route stated in [20] is calculated with an accuracy that is used in applied calculations for $\mathrm{CNC}$ (conventionally with "computer" accuracy), then we obtain the length 567.20 , i.e. Our record route was shorter. In the integer metric used in [20], our route will be one unit longer.

Sample of construction 12. Let us check whether the path of obtaining a record result in certain areas will be shorter if we use scheme (1) for calculations from the same two-member tour with a global index of 0 , as in construction 11 .

And this direction is found. Let us consider the same twomember tour which unites the vertices of 28 66, but with the vertex of embedding 34 , which in the framework of scheme (1) corresponds to the construction from level 084 with a route of 630.94 in length (Fig. 13 on the left).

From it, there are unambiguous constructions of improving routes with zero indices of subsequent levels up to the formation of routes of level 5 . Where it appears after being included in the six-membered tour of vertex 11 (28 34111380866628$), 33$ routes with zero index that duplicate the route of level 40 (Fig. 13 in the center).

From it, there are unambiguous constructions of improving routes with zero indices of subsequent levels up to the formation of routes of level 5. Where appear, after being included in the sixmembered tour of vertex 11 (28 34111380866628$)$ ), 33 routes with zero index that duplicate the route of level 40 (Fig. 13 in the center).
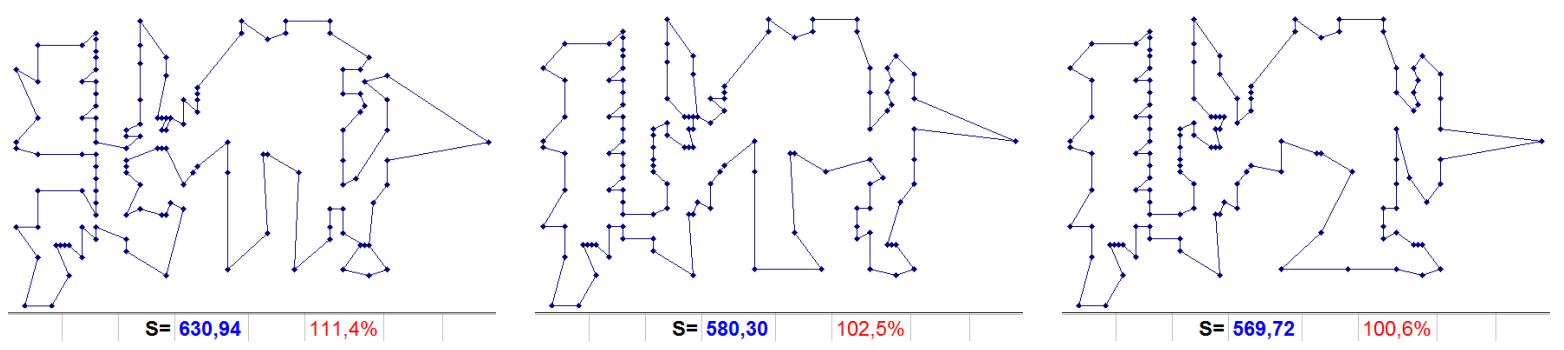

Figure 13. The sequence of key intermediate routes. Additionally, the length and deviation relative to the record are shown.
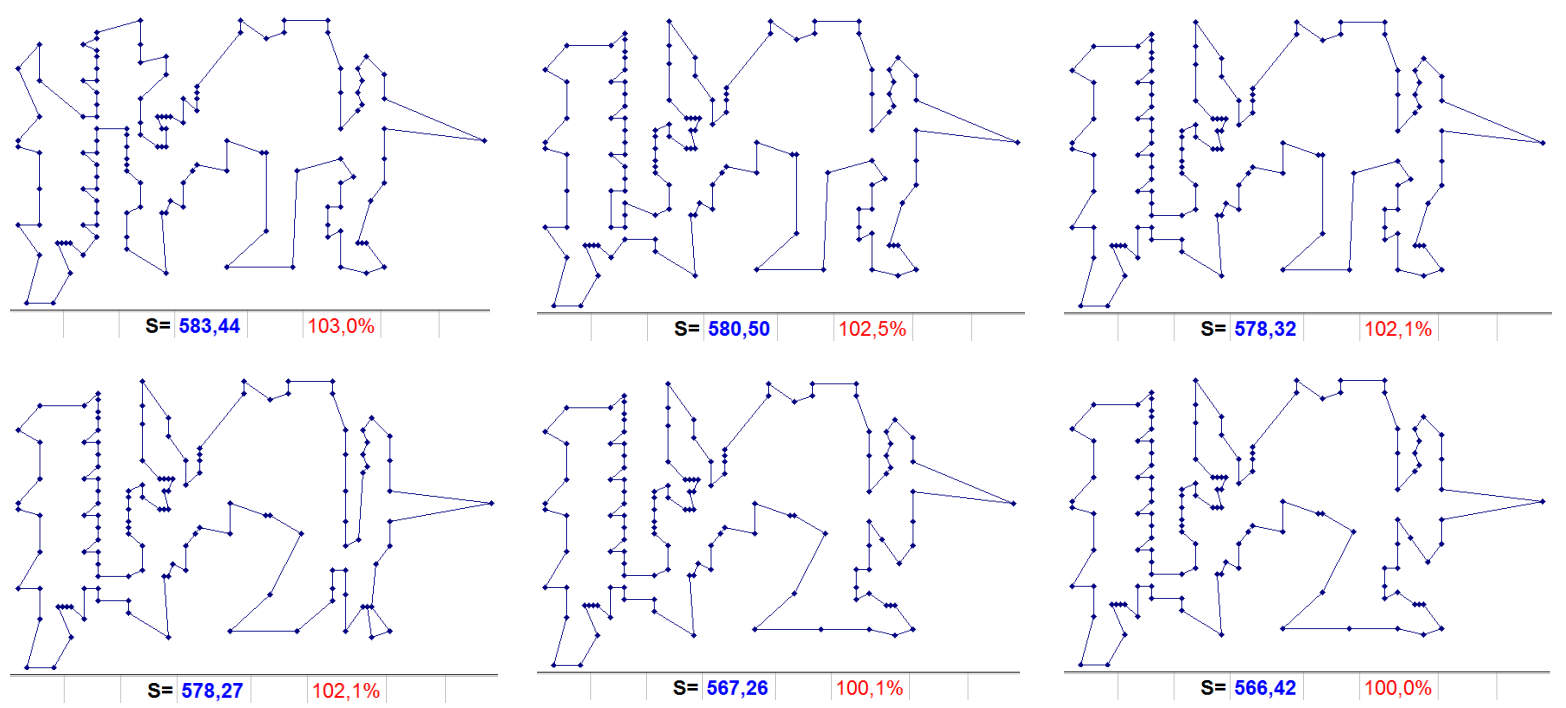

Figure 14. The sequence of key intermediate routes leading to the record. Additionally, the length and deviation relative to the record are shown. 
From one of them (as a result of consecutive unambiguous inclusions in the seven-member tour 2834111380866628 vertices 122106 102), a chain of routes with zero indices of subsequent levels with a minimum length of the resulting route equal to 569.72 is built without branching (Fig. 13 on right). Moreover, there are two such routes and two new directions respectively from the inclusion of vertices 100 and 99 (28 3411 1380861221001061026628 - the first, and 283411138086 122991061026628 - the second). Both directions, in order to reach the record route with a length of 566.42 (Fig. 12 bottom row to the right) assume that the 75 vertex is definitely turned on between 28 and 66, and at the next level 100 - alternative inclusions of the vertex 108 (between 102 and 106).

Sample of construction 13. The record route can be calculated at earlier levels by a repeated number of times and also according to a less laborious scheme (1), if not limited to the calculation, only from the global zero index.

So, for example, among the routes built from all possible combinations of the original two-member tours, the route from the two-member 6666 level 00 with a length of 583.44 (Fig. 14 at the top left) has a global index of 16. It alone determines the inclusion of the 87 in the two-member tour.

Then, there is an unambiguous inclusion in the three-member tour of the vertex 54 (6 8789546 ), after which 9 routes appear with the index 0 level 2 with a length of 580.50 (Fig. 14 in the center above). Among the corresponding vertices of inclusion, only embedding vertices 4 and 112 reduce the route to the next level. Let us track the first of them (6 48789546 ).

From it, there is an unambiguous reduction in the length of the routes to the formation of tours of level 5 , where 8 routes appear with the index 0 , length 578.32 (Fig. 14 at the top right). The corresponding embedding in each of the tours of level 50 again multiplies routes with index 0 of the next level (an average of 8 routes without a change in length). And only embedding already in one of the many tours of level 60 leads to a decrease in the length of the route of level 70 to 578.27 (Fig. 14 at the bottom left).

This reduced route resulted from the inclusion of the vertex 88 in the eight-membered tour ( $\begin{array}{llllll}0 & 11 & 4 & 8788 & 9789 & 54\end{array}$ ). The constructed route predetermines three possible embeddings in the tour 6011487889789546 peaks 9893 or 91 .

Each of these inclusions gives a new reduced route with a length of 567.26 (Fig. 14 in the center below), and, accordingly, 3 initial tours with a unique inclusion of vertex 106 . This inclusion in any of the 3 tours immediately gives a record route (Fig. 14 at the bottom right), which repeatedly manifests itself in different directions when building at level 8 . Thus, up to a record from the initial two-member tour 6896 , it was built on the levels $00-10$ 20 - 30 - 40 - 50 - 60 - 70 - 80 in accordance with the scheme (1).

The record route can be obtained even earlier - at level 70 . With similar constructions according to scheme (1), from a twomember tour, 898789 with a sequence number (lengths of level 00 routes, arranged in ascending order) are approximately midway between the same numbers for "sample build 11 " and "sample build 13".

\section{Conclusion}

A significant number of two-member tours (proportional to the square of the dimension of the problem), practically from each of which with varying degrees of difficulty can be built a record route, at least close to the optimal result, allow us to draw some preliminary conclusions based on the results of a set of calculations.

It can be argued with a high degree of probability that a significant layer of applied problems associated with the efficient operation of $\mathrm{CNC}$ equipment, equivalent in setting to a symmetric traveling salesman problem, can be solved using the method of step-by-step approach to the minimum route.

Moreover, many problems with a dimension of up to 50 elements, close to the present limit of the possibilities of exact methods, can be solved with obtaining an optimal result already at an early stage of calculations. Namely, when calculating at the level of possible combinations of two-member (formation and ranking of routes of zero level) or three-member (formation of routes of level 10) tours.

For problems of greater dimension, especially over 100 elements, it is advisable to reduce the calculations using one of the proposed schemes for organizing calculations (or its modifications). And as experiments show, you can use a more favorable criterion for stopping calculations in the current direction, for example, not threefold (as recommended), but double "repetition of the length of routes". In most cases, this will only lead to a reduction in the directions of calculations leading to a record result, but on the whole, it will accelerate ( $\mathrm{n}$ - fold) the calculation process.

\section{References}

[1] Nikolay Starostin, Konstantin Mironov: Algorithm Modification of the Levelby-Level Approximation to the Minimum Route, 2017 IEEE International Symposium on Signal Processing and Information Technology (ISSPIT), pp. 270-275, December 2017.

[2] N. D. Starostin The method of level-by-level exceptions for optimization of tool movements (in Russian) Collection of materials of the Second International Conference "Intelligent Technologies for Information Processing and Control", Ufa, 2014.

[3] N. D. Starostin. Improvement and detailing of the method of level-by-level exceptions for optimization of tool movements (in Russian) // Information Technologies and Systems [Electronic resource]: tr. Fourth Intern. sci. Conf., Bannoe, Russia, Feb. 25. - March 1, 2015, pp. 33-44

[4] I. I. Melamed The traveling salesman's problem. Exact methods (in Russian) II Melamed, SI Sergeev, I. Kh. Sigal // Avtomat. and telemeh. - 1989. - No. 10. - P. 3-29.

[5] V. V. Borodin Experimental study of the effectiveness of heuristic algorithms for solving the traveling salesman problem (in Russian) VV Borodin, SE Lovetskii, II Melamed, Yu. M. Plotinsky // Avtomat. and Telemeh., 1980, No. 11, 76-84.

[6] Starostin N. D. Finding the shortest path. Accelerated optimization of tool movements - Saarbrucken: LAP LAMBERT Akademic Publishing, 2015. $89 \mathrm{p}$.

[7] N. D. Starostin Variants of calculation of the minimum route of processing (in Russian) Information technologies and systems [Electronic resource]: tr. Fifth International. sci. Conf., Bannoe, Russia, 24 February. - February 28, 2016, pp. $42-48$.

[8] N. D. Starostin Basic variation of the algorithm of level-by-level approximation to the minimal route [Electronic resource, in Russian]: tr. The Sixth Intern. sci. , Bannoe, Russia, March 1 - 5, 2017 P.295-302.

[9] N. D. Starostin, DV Kurennov Level-by-level approach to the minimum route // International scientific and technical conference "Prom-Engineering" in 2017 was held May 16-19 in St. Petersburg. 
[10] MP-TESTDATA - The TSPLIB Symmetric Traveling Salesman Problem Instances - URL: http://elib.zib.de/pub/mp-testdata/tsp/tsplib/tsp. (vizited on 08.02.2016).

[11] Lmatrix Laboratory Featured articles Solutions - URL: http://lmatrix.ru/news/problems (reference date: 01/05/2016)

[12] I. I. Melamed The traveling salesman's problem. Approximate algorithms (in Russian) / II Melamed, SI Sergeev, I. Kh. Sigal // Avtomat. and telemeh. 1989. - No. 11. - P. 3-26

[13] Golden B. L., Skiscim C. C. Using simulated annealing to solve routing and location problems // Nav. Res. Log. Quart. 1986. V. 33. № 2. P. 261-279

[14] National TSP

http://www.math uwaterloo.co/tsp/world/countries.html 05.01 .2017

[15] Yu. A. Kochetov, Computational possibilities of local search in combinatorial optimization, Zh. Vychisl. Math. and Math. fiz. - 2008 - Vol. 48, No. 5 - P. 788-807.

[16] An Interview with Vasek Chvatal. - URL: https://www.cs.rutgers.edu/ mcgrew/Explorer/1.2/\#Farmers (visited on 16.08.2017)
[17] Kochetov Yu., Mladenovich N., Hansen P. Local Search with Alternating Neighborhoods // Discrete Analysis and Operations Research. Series 2. 2003. Volume 10, No. 1. P.11-43.

[18] Metaheuristics: From Design to Implementation. El-Ghazali Talbi. John Wiley \& Sons, 2009, - $624 \mathrm{p}$

[19] R. T. Murzakaev, V. S. Sholov, A. V. Burylov. Application of Metaheuristic Algorithms for minimizing the path of a cutting tool (in Russian) Transactions of PNIPU University on Electrical Engineering, IT, and Control. 2015. №14. C.123-133

[20] VLSI TSP Collection http://www.math.uwaterloo.ca/tsp/vlsi/index.html (дата обращения: 31.08.2017)

[21] MP-TESTDATA - The TSPLIB Symmetric Traveling Salesman Problem Instances - URL: https://people.sc.fsu.edu/ jburkardt/datasets/tsp/tsp.html (дата обращения: 06.02.2018)

[22] In Pursuit of the Traveling Salesman : Mathematics at the Limits ofComputation. Author(s), Cook, William J. Publication, Princeton, NJ : Princeton University Press, 2011. - 245 p. 